\title{
Temperature Dependence of Silicon Luminescence Due to Splitting of the Indirect Ground State*
}

\author{
R. B. Hammond, D. L. Smith, and T. C. McGill† \\ California Institute of Technology, Pasadena, California 91125
}

(Received 28 July 1975)

\begin{abstract}
The temperature dependence of the ratio of LO- to TO-phonon-assisted recombination luminescence of the indirect exciton in silicon is reported. The ratio is found to differ from that observed in absorption and to vary from $\sim 0.3$ at $2^{\circ} \mathrm{K}$ to $\sim 0.1$ at $13^{\circ} \mathrm{K}$. The variation of the ratio with temperature is shown to be due to the splitting of the ground state of the exciton by several tenths of a meV. The relevance of these results to the recombination from the electron-hole condensate in silicon is discussed.
\end{abstract}

The emission and absorption of radiation due to indirect excitons in silicon at low temperatures has been the subject of a number of investigations. $^{1-5}$ Features identified with TA-, LO-, and TO-phonon-assisted transitions have been identified. Dean, Yafet, and Haynes, ${ }^{3}$ Shaklee and Nahory, ${ }^{4}$ and Nishino, Takeda, and Hamaka$\mathrm{wa}^{5}$ have studied the absorption at temperatures less than $10^{\circ} \mathrm{K}$. Shaklee and $\mathrm{Nahory}^{4}$ have correctly interpreted the two features at approximately $1.22 \mathrm{eV}$ as due to absorption with the emission of a LO or TO phonon. These two lines are split by approximately $2 \mathrm{meV}$. Dean, Yafet, and Haynes ${ }^{3}$ have investigated the recombination spectrum at 2.5 and $5.5^{\circ} \mathrm{K}$. They observed two lines at approximately $1.10 \mathrm{eV}$ which they attributed to valley-orbit splitting of the exciton state. Further, they found that the ratio of the relative intensities of these two lines varied with temperature in such a way as to suggest that thermal equilibrium was not established between the two states. Shaklee and Nahory ${ }^{4}$ have pointed out that valley-orbit splitting should not occur and that the two lines observed in luminescence are in fact due to two processes involving the LO and TO phonon, respectively. While this interpretation indicates the origin of the lines, it does not suggest why the ratio of the LO- to TO-phonon process in emission, $\gamma_{E}$, should show the temperature dependence hinted at by the data of Ref. 3 at two temperatures.

In this Letter, we report on a systematic investigation of the temperature dependence of the recombination processes via LO- and TO-phonon emission. We find that the ratio $\gamma_{E}$ varies with temperature in agreement with the two experimental points given by Ref. 3 . This variation of $\gamma$ is shown to come from the fact that the observed LO and TO recombination lines are each made up of two unresolved lines from two exciton states split by less than $1 \mathrm{meV}$. The splitting of the ground state of the exciton into twofold degenerate states, $\Delta_{6}$ and $\Delta_{7}$, is due to the interaction of the hole at $\overrightarrow{\mathrm{K}}=0$ with the anisotropic charge density of the electron in the conduction band. $^{6,7}$ These two states are found to possess different relative rates for TO- and LO-phonon emission in recombination. Hence, a temperature-dependent variation of the relative occupancy of these two exciton states leads to a temperature dependence in $\gamma_{E}$. Analysis of the experimental data gives an energy splitting between the $\Delta_{6}$ and $\Delta_{7}$ states in qualitative agreement with the theoretical values given by McLean and Loudon ${ }^{6}$ and Lipari and Baldereschi. ${ }^{7}$ Although this splitting has been explored in detail theoretically, we believe this is the first experimental observation of the consequences of this splitting in silicon.

The emission spectrum from a sample of laserexcited silicon is shown in Fig. 1. The silicon crystal was high-purity $p$ type with a net impurity concentration $N_{A}-N_{D} \sim 2 \times 10^{11} \mathrm{~cm}^{-3}$. The spectrum was obtained by illuminating the sample with a GaAs laser which produced pulses of $2 \mu \mathrm{sec}$ duration with a repetition rate of $20 \mathrm{kHz}$. The optical power was approximately $3 \mathrm{~W}$. The luminescence was analyzed with a Spex 1400-II spectrometer, detected with an RCA 7102 S-1 photomultiplier tube operated at $195^{\circ} \mathrm{K}$, and processed with a lock-in amplifier. The spectrum was independent of laser power and hence showed no evidence of heating.

The luminescence line shape was fitted with the expression proposed by Elliott. ${ }^{8}$ The intensity was assumed to vary as

$$
I(E) \sim\left(E-E_{x}\right)^{1 / 2} \exp \left[-\left(E-E_{x}\right) / k_{\mathrm{B}} T\right],
$$

where $E_{x}$ is the threshold energy. This theoretical expression for the intensity was modified to include the effects of detector sensitivity, spec- 


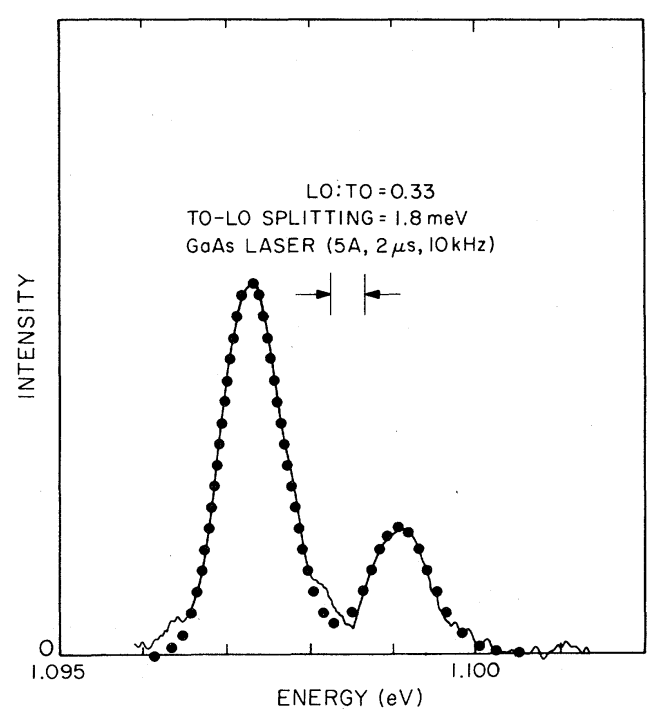

FIG. 1. Luminescence spectrum of silicon at $2.1^{\circ} \mathrm{K}$. The silicon was excited using a GaAs laser. The solid line shows the experimental results. The TO-assisted recombination produces the line centered at $\sim 1.097 \mathrm{eV}$. The LO-assisted recombination produces the line centered at $\sim 1.099 \mathrm{eV}$. The dots are the theoretical line shape produced by the procudure described in the text.

trometer transmission and broadening, and a Gaussian broadening due to phonon lifetime.

The theoretical curve in Fig. 1 was obtained using this procedure with energy broadening due to phonon lifetime characterized by a standard deviation of $0.34 \mathrm{meV}$; an energy separation between the TO and LO lines of $1.8 \mathrm{meV}$; a temperature of $2.1^{\circ} \mathrm{K}$, the bath temperature; and $\gamma_{E}$ of 0.33 . The value of the broadening used here is in agreement with the value deduced from derivative absorption spectroscopy. ${ }^{5}$

Spectra at other temperatures exhibit similar features except that as temperature is increased the separated lines tend to merge. The values of $\gamma_{E}$ at other temperatures were obtained by the same line-fitting procedure. The results of these experiments along with the results reported by other authors for emission and absorption ${ }^{3,5}$ are given in Fig. 2. These results show that $\gamma_{E}$ varies from $\sim 0.3$ at $2.1^{\circ} \mathrm{K}$ to $\sim 0.1$ at $13^{\circ} \mathrm{K}$. The data also show that $\gamma_{E}$ approaches the ratio for absorption $\gamma_{A}$ at higher temperatures.

The variation of $\gamma_{E}$ with temperature may be understood in terms of the splitting of the groundstate exciton level into two levels separated by an energy $\Delta E$. The ratio $\gamma_{E}(T)$ is given by

$$
\gamma_{E}(T)=\frac{R_{\mathrm{LO}} \Delta_{6}+R_{\mathrm{LO}} \Delta_{7} \exp \left(-\Delta E / k_{\mathrm{B}} T\right)}{R_{\mathrm{TO}} \Delta_{6}+R_{\mathrm{TO}} \Delta_{7} \exp \left(-\Delta E / k_{\mathrm{B}} T\right)},
$$

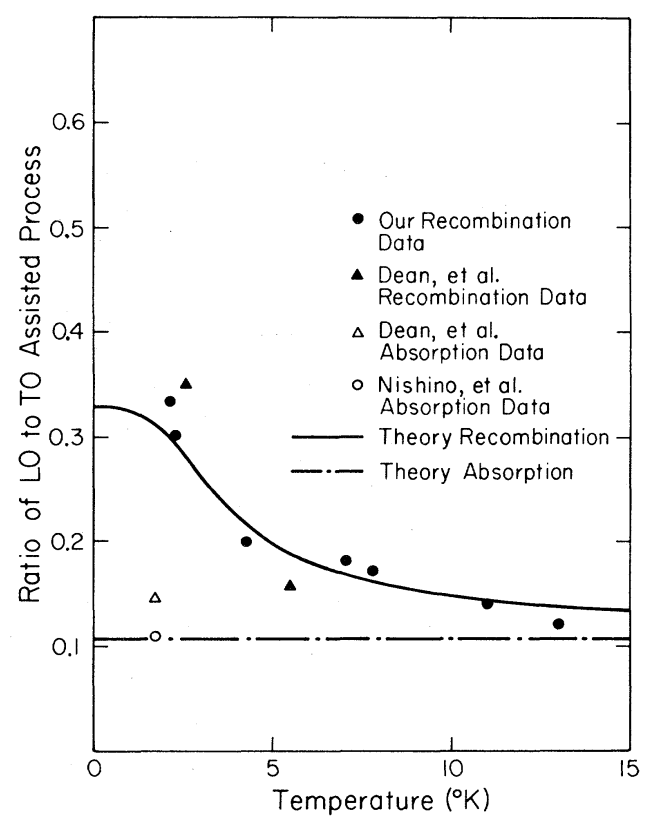

FIG. 2. The ratio of LO- to TO-phonon-assisted emission and absorption as a function of temperature. The experimental data shown by $\Delta$ and $\Delta$ are from Ref. 3; those shown by $O$, from Ref. 5 . The theoretical curves are obtained by taking $\Delta E=0.6 \mathrm{MeV}$ and, with $A$ positive, $A=2.2$ and $B=1.3$, or with $A$ negative, $A=-0.4$ and $B=0.2$. Both of these sets of values produce identical curves.

where $R_{\mathrm{LO}}{ }^{\Delta_{6}}$ and $R_{\mathrm{LO}}{ }^{\Delta_{7}}$ are the rates for photon emission via LO-phonon emission for the more tightly bound state $\Delta_{6}$ and the split-off excited state derived from the ground state $\Delta_{7}$, respectively. $R_{\text {TO }}{ }^{\Delta_{6}}$ and $R_{\text {TO }}{ }^{\Delta_{7}}$ are defined in an analogous way for the TO-phonon-assisted process. In absorption the ratio $\gamma_{A}$ is given by the ratio of the sum of the rates. For high temperatures such that $k_{\mathrm{B}} T \gg \Delta E$, we have that $\gamma_{E}(T)$ approaches $\gamma_{A}$ in agreement with experiment.

From Eq. (2) it is clear that to predict $\gamma_{E}(T)$, we must know the ratios of the various rates. As illustrated in Fig. 3, the two most likely recombination paths are as follows: (1) The electron emits a phonon and makes a transition from the $\Delta_{1}$ state in the conduction band to the $\Gamma_{15}{ }^{-}$ state in the conduction band and then recombines with a hole in either the $\Delta_{6}$ state in the case of the more tightly bound exciton or the $\Delta_{7}$ state in the case of the less tightly bound exciton. The process is allowed for TO-phonon emission only.$^{9}$ (2) The electron in $\Delta_{5}$ emits a phonon and recombines with a hole in the state $\Delta_{6}$ or $\Delta_{7}$, respectively. Then the electron in state $\Delta_{1}$ in the con- 
duction band recombines with the hole in $\Delta_{5}$, emitting a photon. When the hole is in the $\Delta_{6}$ state, this process is allowed for the LO phonon only, and is TO- and LO-phonon allowed when the hole is in the $\Delta_{7}$ state. A group-theoretical study of the relative rates ${ }^{10,11}$ using an analysis similar to that used by Lax and Hopfield ${ }^{9}$ leads us to conclude that $\gamma_{E}(T)$ must be of the form

$$
\gamma_{E}(T)=|B|^{2}\left[\frac{3+\exp \left(-\Delta E / k_{\mathrm{B}} T\right)}{3|A|^{2}+\left[4|A+1|^{2}+|A|^{2}\right] \exp \left(-\Delta E / k_{\mathrm{B}} T\right)}\right],
$$

where $A$ and $B$ involve ratios of energy denominators from the second-order perturbation theory, the reduced matrix elements ${ }^{12}$ of the momentum operator $p$, and the TO and LO part of the electron-phonon interaction Hamiltonian $H_{e p}{ }^{\mathrm{TO}}$ and $H_{e p}{ }^{\mathrm{LO}}$, respectively:

$$
A=\left(\frac{\Delta E_{v}}{\Delta E_{c}}\right)\left(\frac{\left\langle\Gamma_{25}^{+}\|p\| \Gamma_{15}{ }^{-}\right\rangle\left\langle\Gamma_{15}{ }^{-}\left\|H_{e p}{ }^{\mathrm{TO}}\right\| \Delta_{1}\right\rangle}{\left\langle\Delta_{5}\|p\| \Delta_{1}\right\rangle\left\langle\Gamma_{25}{ }^{+}\left\|H_{e p}{ }^{\mathrm{TO}}\right\| \Delta_{5}\right\rangle}\right),
$$

where $\Delta E_{v}$ and $\Delta E_{c}$ are the energy denominators for transitions through the $\Delta_{5}$ and $\Gamma_{15}{ }^{-}$states, respectively; and

$$
B=\frac{\left\langle\Gamma_{25}{ }^{+}\left\|H_{e p}{ }^{\mathrm{LO}}\right\| \Delta_{5}\right\rangle}{\left\langle\Gamma_{25}{ }^{+}\left\|H_{e p}{ }^{\mathrm{TO}}\right\| \Delta_{5}\right\rangle} .
$$

Since the space group for the diamond lattice contains the inversion symmetry and the states must be invariant under time reversal, then $A$ and $B$ may be taken to be real. ${ }^{13}$ The sign of $B$ is irrelevant in Eq. (3) but the sign of $A$ is important. The exact values of $A$ and $B$ cannot be obtained from our analysis but we expect $A$ and $B$ to be of order unity. Regardless of the values of $A$ and $B$, Eq. (3) shows that $\gamma_{E}$ must decrease with increasing temperature.

The comparison between the experimental re-

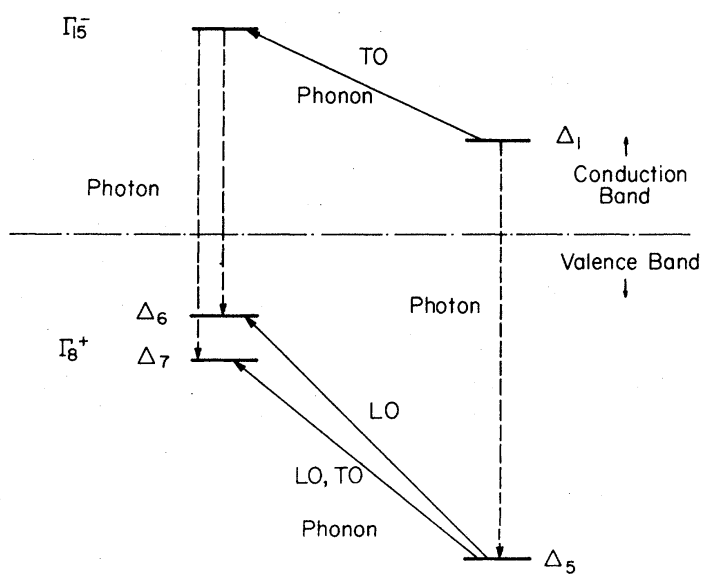

FIG. 3. Schematic diagram of the two recombination processes in silicon. The splitting of the ground state of the exciton is shown in the hole band where the fourfold degeneracy of the $\Gamma_{8}{ }^{+}$states is split into the two twofold degenerate states $\Delta_{6}$ and $\Delta_{7}$ which are labeled according to the irreducible representations of the group of $\overrightarrow{\mathrm{K}}$ for $\overrightarrow{\mathrm{K}}$ along the $\Delta$ direction. sults and theoretical results for both emission and absorption is contained in Fig. 2. The values of $\Delta E, A$, and $B$ were determined by a leastsquares fit to the experimental data, excluding that obtained from Dean, Yafet, and Haynes. ${ }^{3}$ The value of $\Delta E$ which produced the best fit is $0.6 \mathrm{meV}$. The values of $A$ and $B$ are not uniquely determined by this procedure; either $A=2.2$ and $B=1.3$ or $A=-0.4$ and $B=0.2$ produce the same curve. The theoretical curves for $\gamma_{E}$ and $\gamma_{A}$ for these parameters are shown in the figure. The agreement between the experimental results and theoretical calculations is quite good. The value of $\Delta E$ taken here is in good agreement with the calculations of McLean and Loudon. ${ }^{6}$ However, the agreement is not greatly changed for a range of values of $\Delta E$ between 0.3 and $0.7 \mathrm{meV}$. While we are unable to give a precise value for the splitting, the good agreement between theory and experiment provides the first experimental confirmation of the splitting of the ground state of the exciton in silicon.

The luminescence from the electron-hole condensate in silicon also possesses a strong line due to LO- and TO-phonon-assisted recombination. ${ }^{14-16}$ In this case it is not possible to resolve the two lines since the intrinsic width of the condensate line, approximately $12 \mathrm{meV}$, is greater than the splitting between the TO- and LO-assisted lines. However, in fitting the condensate line shape, it is essential to have a value for the ratio of these two processes. ${ }^{16}$ Since holes in the condensate should occupy the states $\Delta_{6}$ and $\Delta_{7}$ with equal probability, the correct value of the ratio of LO to TO processes is the value observed in exciton absorption, $\gamma=0.11$.

The authors gratefully acknowledge an essential discussion with J. J. Hopfield and many useful discussions with J. W. Mayer. 
*Work supported in part by the U. S. Office of Naval Research under Contract No. N00014-67-A-0094-0036 and the U.S. Air Force Office of Scientific Research under Grant No. 73-2490.

tAlfred P. Sloan Foundation Fellow.

${ }^{1}$ G. G. MacFarlane, T. P. McLean, J. E. Quarrinton, and V. Roberts, Phys. Rev. 111, 1245 (1958).

${ }^{2}$ J. R. Haynes, M. Lax, and W. F. Flood, J. Phys. Chem. Solids $\underline{8}, 392$ (1959).

${ }^{3}$ P. J. Dean, Y. Yafet, and J. R. Haynes, Phys. Rev. 184, 837 (1969).

${ }^{4}$ K. L. Shaklee and R. E. Nahory, Phys. Rev. Lett. 24, 942 (1970).

${ }^{5}$ T. Nishino, M. Takeda, and Y. Hamakawa, Solid State Commun. 12, 1137 (1973).

${ }^{6}$ T. P. McLean and R. Loudon, J. Phys. Chem. Solids 13,1 (1960).

${ }^{7}$ N. O. Lipari and A. Baldereschi, Phys. Rev. B $\underline{3}$, 2497 (1971).

${ }^{8}$ R. J. Elliott, Phys. Rev. 108, 1384 (1957).

${ }^{9}$ M. Lax and J. J. Hopfield, Phys. Rev. 124, 115
(1961).

${ }^{10}$ D. L. Smith and T.C. McGill, to be published.

${ }^{11}$ In deriving Eq. (3), we have neglected differences in the exciton dispersion relations for the two exciton states. See E. O. Kane, Phys. Rev. B 11, 3850 (1975).

${ }^{12}$ G. F. Koster, J. O. Dimmock, R. G. Wheeler, and H. Statz, Properties of the Thirty-Two Point Groups (M.I.T. Press, Cambridge, Mass., 1963), p. $13 \mathrm{ff.}$

${ }^{13}$ E. I. Blount, in Solid State Physics, edited by F. Seitz and D. Turnbull (Academic, New York, 1962), Vol. 13, p. 359 ff.

${ }^{14}$ J. R. Haynes, Phys. Rev. Lett. 17, 860 (1966).

${ }^{15}$ Ya. E. Pokrovsky, A. Kamisky, and K. Svistunova, in Proceedings of the Tenth International Conference on the Physics of Semiconductors, Cambridge, Massachusetts, 1970, edited by S. P. Keller, J. C. Hensel, and F. Stern, CONF-700801 (U. S. Atomic Energy Commission, Division of Technical Information, Springfield, Va., 1970), p. 504 .

${ }^{16}$ R. B. Hammond, T. C. McGill, and J. W. Mayer, to be published.

\title{
COMMENTS
}

\section{Fine-Structure Corrections and Electromagnetic Decays of Charmonium*}

\author{
J. Pumplin, W. Repko, and A. Sato $\dagger$ \\ Department of Physics, Michigan State University, East Lansing, Michigan 48824
}

(Received 4 August 1975)

\begin{abstract}
We calculate fine-structure corrections to the spectrum of $\psi / J$ particles in the charmonium model, assuming that the linear potential which confines the quarks is due to an effective vector exchange. We also calculate the electromagnetic decay rates between the various low-lying $s$ and $p$ states.
\end{abstract}

Recent observations of monoenergetic $\gamma$ rays in colliding-beam experiments at DESY ${ }^{1}$ and at Stanford Linear Accelerator Center ${ }^{2}$ lend support to the charmonium model ${ }^{3}$ of the narrow resonances $\psi(3.1)$ and $\psi(3.7)$. In particular, the prediction ${ }^{4.5}$ of three $p$ states, with masses around $3.5 \mathrm{GeV}$, and the associated $\gamma$-ray decay schemes, could form a basis for understanding the data. A measurement of the spacing between the ${ }^{3} P_{0},{ }^{3} P_{1}$, and ${ }^{3} P_{2}$ levels - assuming they are found-will yield information about the quark-antiquark $(c \bar{c})$ dynamics. Therefore, we have calculated these fine-structure splittings, under the assumption that the two-body system is essentially nonrelativistic ${ }^{3}$ and that the binding results from an effective vector exchange which provides quark confinement.

Specifically, we assume an effective $c \bar{c}$ interaction in momentum space of the form

$$
I=\left(\gamma_{4} \gamma_{\mu}\right)^{(1)}\left(\gamma_{4} \gamma_{\mu}\right)^{(2)} \bar{v}\left(k^{2}\right)
$$

where $k$ is the four-momentum transfer and $\bar{v}\left(k^{2}\right)$ is a confining potential. In the nonrelativistic limit, where $k_{0} \rightarrow 0$, the Fourier transform of $\bar{v}\left(\overrightarrow{\mathrm{k}}^{2}\right)$ is $v(r)$, the potential used in the two-body Schrödinger equation. To obtain the lowest-order relativistic corrections to $v(r)$, we expand Eq. (1) to order $(\nu / c)^{2}$ and express the result in nonrelativistic (two-component) form. We then transform to coordinate space 